7. Ferrum oxydatum saccharatum (H. EPPINGER) wird nach der Turnbull and Perls-Reaktion beurteilt in mittleren Gaben von der Bauchhöhle der weißen Maus aus vorwiegend im Netz, in den periportalen und peribronchialen Lymphdrüsen und in der Milz, fast gar nicht in der Leber und Lunge und den übrigen Organen gestapelt (vgl. dazu auch STRASSER). Solche Tiere sind weitgehend geschützt gegen intraperitoneale, nicht gegen subcutane Fermentvergiftung. Das zeigt, daß neben der Leber auch der Milz eine wichtige Rolle bei diesem Schutze zufällt.

8. Der durch Tusche, Pyrrholblau und kolloidales Eisen gewährte Schutz richtet sich gegen das Ferment selbst, nicht aber gegen seine Verdauungsprodukte. Er ist unwirksam bei der intraperitonealen Peptonvergiftung.

9. Von anderen als Eiweißzerfallsvergiftung aufgefaßten Formen haben wir bisher die Hämolysinvergiftung am Beispiel des Rinderserums und der weißen Maus erprobt. Auch hier bestand ein weitgehender. Schutz gegen tödliche Gaben von der Bauchhöhle aus (vgl. dazu die jüngsten Angaben von H. Eppinger (Wien. klin. Wochenschr. I922 und von BrELING und IsAaC in dieser Zeitschrift). Hingegen versagte die Vorbehandlung sowohl am Beispiele der einfachen Retentionsurämie des Kaninchens, entsprechend dem wesensgleichen Versagen gegen Pepton, als auch beim photodynamischen Lichttode (weiße Maus) und bei Hautverbrühungen (Meerschweinchen). Das Letzterwähnte kann nicht als Beweis gegen die innere Zusammengehörigkeit dieser Vergiftungsformen untereinander und mit der Fermentvergiftung gewertet werden, da gerade hier für den Angriff des Giftes ähnliche Verhältnisse gegeben sind, wie bei unserer Fermentvergiftung von der Unterhaut aus, wo gleichfalls ein Schutz nicht wahrgenommen wurde.

Die Einzelheiten unserer Versuche, eine Würdigung der vorliegenden Literatur über Fermentvergiftungen und über den reticulo-endothelialen. Apparat, sowie die Ausblicke, die sich auf eine Verwendung dieses Schutzes gegen spontane Fermentvergiftungen von der Bauchhöhle aus ergeben, behalten wir einer ausführlichen Widergabe in einer Fachzeitschrift vor. Das soll nach Abschluß von Beobachtungen geschehen, welche den EinfluB solcher Stapelung auf den anaphylaktischen Schock zum Gegenstande haben.

Die Untersuchungen werden ausgeführt mit Hilfe einer Unterstützung von W. F. Petersen, Chicago, wofür schon an dieser Stelle gebührender Dank gesagt werden möge.

\section{NEUROGENE HETEROCHROMIE DER IRIS, EIN SYMPTOM INNERER KRANKHEITENI).}

Voll

Dr. Friedrich KaUfFMANN,

Assistenzarzt der Medizinischen Universitätsklinik Frankfurt a. M. (Direktor: Prof. G. v. BERGMANN.)

Daß Erkrankungen der Visceralorgane auf $z$. T. weit entfernte Körpergebiete einen $\operatorname{Einflu} B$ ausüben können, ist eine bekannte Tatsache, welche allerdings die ihr gebührende Würdigung nicht immer zu erfahren scheint. Außer den mannigfachen Erscheinungen visceromotorischer, viscerosensibler und viscerovisceraler Reflexe, wie sie z. B. für die Erkrankungen der Gallenblase v. BERGMANN ${ }^{2}$ ) kürzlich zusammenfassend ausführlich dargestellt hat, kann auch in vielen derartigen Fällen eine Erweiterung der rechten Pupille ein wertvoller diagnostischer Hinweis sein, welcher oft sogar im Latenzstadium einer Cholecystitis bzw. eines Gallensteinleidens verwertbar ist. Auch bei der Pyelitis ist häufig das gleiche Phänomen vorhanden. Solche auf "Irradiation" beruhende Fernsymptome geben dem Einzelfall ein diagnostisches Gepräge, welches am Krankenbett täglich Anregung liefert und im Erkennen und Auffassen des Individuellen im Krankheitsbilde förderlich ist (v. BERGMANN).

1) Nach einem am 33 . Juni $x 922$ im Biologischen Verein in Frankfurt a. M. gehaltenen Vortrag.

3) G. v. BERGMANN, Neuere Gesichtspunkte bei der Gallenblasenkrankheit. Jahresk. f. ärztl. Fortbild. Märzheft I922, S. 7 .
So möchte ich in den folgenden Ausführungen die Aufmerksamkeit auf ein weiteres Fernsymptom lenken: auf Unterschiede in der Pigmentierung der Iris.

Seitdem ich auf dieses Symptom achte, habe ich eine ganze Reihe von Patienten gesehen, bei denen es sich um kongenitale oder durch entzündliche Prozesse bedingte Unterschiede im Irispigment handelte. Von solchen Fällen soll hier nicht die Rede sein. Die folgenden Ausführungen knüpfen vielmehr an eine Anzahl von Patienten mit verschiedenen inneren Krankheiten an, in deren Verlauf Erscheinungen eines einseitig gesteigerten Reizzustandes im Gebiete des Halssympathicus aufgetreten waren. Als Symptom dessen fand sich neben einseitiger Erweiterung der Pupille in einer Reihe von Fällen Neigung zu halbseitigem Schwitzen. Asymmetrie des Gesichtes infolge Atrophie der Gesichtsmuskulatur und alle diese Patienten ließen eine Differenz in dem Pigmentgehalt der rechten und linken Iris erkennen. Diese klinische Beobachtung legte die Frage nahe, ob nicht die Differenz in der Pigmentierung der Iris auf die gleiche Ursache wie die übrigen erwähnten Symptome, nämlich auf den krankhaft gesteigerten Reizzustand des gleichseitigen Halssympathicus zu beziehen sei. Vier der betreffenden Patienten gaben mit Sicherheit an, in der Jugend "gleichfarbige Augen" gehabt zu haben. Wenn wir außerdem berücksichtigen, daß Experimente von Bistis (s. später) Beziehungen zwischen Sympathicus und Irisfarbe sichergestellt haben, so scheint es nicht unberechtigt zu sein, in alien hier kurz mitzuteilenden Fällen, in denen Residuen früher überstandener lokaler Augenkrankheiten nicht nachgewiesen werden konnten, die Hetercchromie als erworben anzusehen und in ihr ein Symptom einseitig gesteigerten Reizzustandes im Halssympathicus zu erblicken.

Zum ersten Male sah ich eine Heterochromie bei einem 3ojährigen Patienten mit intra partum entstandener rechtsseitiger Klumpke scher Lähmung und Horner schem Symptomenkomplex. Neben den bekannten Erscheinungen der Myosis, Ptosis und geringem Enophthalmus bestand eine deutliche Atrophie der rechtsseitigen Gesichtsmuskulatur, ferner vasomotorische Störungen, indem die rechte Gesichtshälfte in der Regel stärker gerötet war als die linke. Außerdem starkes rechtsseitiges Schwitzen. Farbe der Iris: Rechts reinblau, links hellbraun. Die in der Angenklinik (Dr. METZGER) vorgenommene Untersuchung an der Spaltlampe ergibt rechts nahezu vollkommenes Fehlen des Pigmentes im Irisstroma, auch das hintere Pigmentblatt scheint rechts geringer ausgebildet $z u$ sein als links, wie die dürftige Pigmenteinfassung des Pupillarsaumes zeigt. Kein Zeichen einer durchgemachten Uveitis; kein Unterschied in der Pigmentierung des Augenhintergrundes.

Im AnschluB an diesen Fall habe ich mehrere Patienten beobachtet, bei denen ein einseitig erhöhter Reizzustand im Gebiet des Halssympathicus mit einer Differenz im Pigmentgehalt der Iris verbunden war. Darunter befanden sich 4 Kranke mit tuberkulöser Frkrankung einer Lungenspitze: neben Erweiterung der gleichseitigen Pupille war eine geringere Pigmentierung der Iris des gleichen Auges deutlich zu erkennen.

$\mathrm{Zu}$ dieser Gruppe gehört z. B. ein 29jähriger Arzt (Dr. O.), welcher im Jahre I9 Io eine linksseitige trockene Pleuritis, im Jahre 19I I eine linksseitige Hilusdrüsentuberkulose mit Affektion der linken Spitze überstand und jetzt klinisch geheilt ist. Im Röntgenbild ist die linke Spitze leicht verschattet und etwas kleiner als die rechte. Die rechte Iris ist graugrün gefärbt, während die linke, welche bei mittlerer Beleuchtung eine Spur weiter ist, fast rein graublaue Farbe zeigt. Diese Differenz in der Farbe der Augen hat in der Jugend mit Sicherheit nicht bestanden.

Bei 2 Patienten mit Aorteninsuffizienz und Erweiterung der Aorta ascendens fand sich neben einer geringen Erweiterung der rechten Pupille eine analoge Veränderung: beide zeigten rechts eine reinblaue Irisfarbe, links eine blaugrüne, bzw. branne Farbe. Auch in diesen Fällen hat die ungleiche Färbung der Augen in der Kindheit nicht bestanden.

Ferner sah ich eine Kranke, welche seit mehreren Jahren an wiederholt aufgetretenen Gallensteinanfällen verbunden mit heftigen rechtsseitigen Kopfschmerzen litt. Mit hochgradigem Ikterus wurde die Patientin in die Klinik eingeliefert. Die rechte Pupille ist erheblich weiter als die linke, 
die rechte Iris fast rein graublau, während die linke eine hellbraune Färbung zeigt. Anfangs lag die Vermutung nahe, daß in diesem Falle die Heterochromie auf verminderte Ablagerung von ikterischem Pigment in der rechten Iris zurückzuführen sei. Später aber, als nach der Operation (Steine in der Gallenblase und im Choledochus) der Ikterus nicht mehr bestand, war die Differenz der. Irisfarbe in gleicher Weise erkennbar. Es dürfte folglich die Depigmentierung der rechten Iris wie die Erweiterung der Pupille auf den chronisch bestehenden und reflektorisch bedingten Reizzustand des rechtsseitigen Sympathicus zurückzuführen sein.

Eine 37jährige Patientin möchte ich ferner anführen, bei welcher seit Jahren heftige linksseitige Migräneanfälle auftraten. Es bestanden auch tubare Beschwerden infolge Erkrankung der linken Nebenhöhlen. Die linke Gesichtshälfte fand sich fast stets stärker gerötet als die rechte. Neben Erweiterung der linken Pupille ist die Farbe der linken Iris graublau, die der rechten braun. Dieser Unterschied in der Farbe der Augen, welche in der Jugend nicht bestanden hat, soll im Jahre IgI6 von einem Arzt zuerst festgestellt worden sein.

Bei allen Kranken war also die Iris der dem Sitz der Krankheit entsprechenden Seite die hellere. Es scheint daher die SchluBfolgerung nahezuliegen, daß chronisch erhöhter Reizzustand im Halssympathicus, sei dieser nun direkt ausgelöst oder auf reflektorischem Wege von inneren Organen her bedingt, zu einer Verminderung des Irispigmentes führen kann.

Die Mehrzahl der Augenärzte nimmt heute offenbar an, daß jede Heterochromie der Iris entweder angeboren oder die Folge chronisch entzündlicher Prozesse sei. Auch HEINE ${ }^{1}$ ) scheint diesen Standpunkt neuerdings noch zu vertreten. Und doch liegen in der Literatur einzelne experimentelle Untersuchungen vor, in welchen nachgewiesen werden konnte, daß Durchschneidung des Halssympathicus im Tierversuch nach Ablauf von 2-5 Monaten zu Entfärbung der gleichseitigen Iris führen kann. Uber solche Versuche, welche den Einfluß des sympathischen Nervensystems auf die Pigmentierung sicherstellen dürften, hat wohl Bistis ${ }^{2}$ ) als erster berichtet. Er hat auch bereits histologische Befunde an der veränderten Iris mitgeteilt. Unter Hinweis auf frühere Autoren, wie Lutz, Mayon, GalEzowsKY, welche an dem helleren Auge Lähmungserscheinungen des Halssympathicus feststellen konnten, wirft Bistis die Frage auf, ob nicht in manchen Fällen als Ursache der Heterochromie eine Lähmung des Halssympathicus $\mathrm{zu}$ betrachten sei.

Abgesehen von dem ersten hier mitgeteilten Fall von KIUMPKE scher Lähmung mit HoRNerschem Symptomenkomplex handelt es sich nun freilich bei unseren Kranken nicht um Lähmung, sondern um chronische Reizung des Halssympathicus. Es könnte auffallend erscheinen, da $\beta$ beide Zustände zu der gleichen Veränderung im Pigmentstoffwechsel der Iris im Sinne einer Atrophie führen sollten. Demgegenüber ist jedoch darauf hinzuweisen, daß dies für andere Gewebe eine wohlbekannte Erscheinung ist. So ist z. B. die peripherisch-neurotische Knochenatrophie nach den Anschauungen GoLDSCHEIDERs ${ }^{3}$ ) die Folge eines krankhaft gesteigerten nervösen Reizzustandes. In ähnlicher Weise, wie dies vorher schon CASSIRER ${ }^{4}$ ), zu allererst wohl CHARCOT ausgesprochen hat, gibt GoLDSCHEIDER der Meinung Ausdruck, $\mathrm{daB}$ anscheinend ganz allgemein für trophische Störungen nicht so sehr die Aufhebung der Innervation, als vielmehr ein Izrankhaft gesteigerter nervöser Reizzustand als Ursache in Frage Lommen dürfte." Besteht unsere Auffassung von der Atiologie der Depigmentierung in den hier mitgeteilten Fällen zu Recht, daß sie nämlich zurückzuführen ist auf chronische Reizung des Halssympathicus, so würden diese Beobachtungen eine weitere Stütze für die CASSIRER-GoldSCHEIDER sche Anschauung sein.

1) L. HEINE, Augenveränderungen bəi inneren Krankbeiten. Berlin, Jui. Springex I 921 .

2) I. BISTIS, Klinische und experimentelle Untersuchungen über die Ätiolog.e dar Heterochromie. Arch, f. Augenheilk, 75, 302; rgr3.

8) A. GOLDSCHEIDER, Übær neurotische Knochenatrophie usw. Zaitschr. f. klin. Mied. $60, \mathrm{I}, \mathrm{rg} 05$.

a) CASSIRER, Die vasomot. trophisçien Neurojen, I. Aufl. rgor, S, 97.
Beobachten wir also eine Heterochromie der Iris und wollen wir ihre Ätiologie klären, so werden wir neben der Möglichkeit kongenitaler Entstehung nicht nur auf Residuen lokaler Augenkrankheiten zu achten haben, sondern wir werden auch den ,neurogenen Ursprung der Heterochromie" in dem hier mitgeteilten Sinne berücksichtigen müssen.

Andererseits wird uns in Zukunft als Fernsymptom innerer Krankheiten nicht nur eine Differenz in der Weite der Pupillen interessieren, sondern wir werden unser Augenmerk auch auf Unterschiede im Pigmentgehalt der Iris richten können. In manchen Fällen wird es dann berechtigt sein, beide Symptome auf die gleiche Ursache, auf direkte oder reflektorisch bedingte Reizung đes Halssympathicus zurückzuführen. Wenn freilich die Heterochromie bei weitem nicht in allen Fällen vorhanden ist, in denen eine Anisocorie auf der erwähnten Grundlage besteht, so wird uns das verhältnismäßig seltene Vorkommen neurogener Pigmentunterschiede im Irisstroma ein Hinweis dafür sein, daß wir für ihr Zustandekommen noch Faktoren anzunehmen haben, die uns vorläufig unbekannt sind und die das Individuelle im Krankheitsbilde ausmachen.

\section{ÜBER DIE BEDEUTUNG UND DEN WERT VON NÄHRKLISTIEREN BEIM SÄUGLING.}

Von

Dr. W. LASCH, Assistenzarzt.

Aus dem Waisenhaus und Kinderasyl der Stadt Berlin.

(Leitender Arzt Prof. Dr. L. F. MEYER.)

Es gibt wohl kaum eine schwierigere ärztliche Aufgabe als die Behandlung einer akuten Ernährungsstörung bei einem atrophischen Säugling. Auf der einen Seite zwingt die Verdauungsstörung zur Schonungsdiät für den Darm und zu vorübergehendem Hunger, auf der anderen Seite kann der abgemagerte und jeder Reserve beraubte Organismus auch kürzeste Hungerpausen kaum noch ertragen, und der Nahrungsentziehung folgt nicht selten Kollaps und tödlicher Verfall. Wer nur die Darmerscheinung in den Brennpunkt der Behandlung stellt und hungern läßt, schädigt unmittelbar den Gesamtorganismus, und wer den Bedürfnissen des Organismus einseitig Rechnung trägt und erzährt, wird die Magendarmerscheinungen nicht zur Besserung bringen und auf diesem Weg mittelbar denselben Schaden stiften. So zwischen Scylla und Charybdis schwankend, begrüßt man jedes Mittel, das auch nur um ein weniges die drohenden Gefahren mildern kann. Ein solches Mittel sind die vor kurzem von GöPPERT empfohlenen Zuckerklistiere.

Von dem Gedanken ausgehend, daß Nahrungsstoffe, die mit Umgehung des Dünndarms eingeführt werden, dem hungernden Organismus zugute kommen, ohne die Gefahr der pathologischen Gärungsvorgänge im Dünndarm zu vermehren, benutzte GöPPERT die seit langem bekannte Resorptionsfähigkeit des Dickdarms, indem er den Kindern per rectum 5-6 mal täglich in Mengen von 70 bis $100 \mathrm{~g}$ IO-I $5-20$ proz. Lösungen von ,Maltose, besonders in der leicht erhältlichen Form des Nährzuckers und der Nährmaltose " instilliert. GöPPERTS Erfahrungen zeigten in der Tat, daß die Zuckerklistiere in diesen Stadien der Ernährungsstörung praktischen Erfolg hatten. Es gelang ihm, intoxizierte, schwer atrophische Kinder, die erfahrungsgemäß häufig wenige Stunden nach Beginn der Teediät infolge des Hungers unter Krämpfen. kollabierten, durch Anwendung von. Nährklistieren am Leben zu erhalten. Auch in anderen Fällen, in denen die Ernährung per os auf Schwieriglkeiten stieß, bei Kindern mit. Pylorusstenose, stark herabgesetztem Bewußtsein, Kollaps, Appetitlosigkeit, Erbrechen, leisteten ihm die Einläufe wertvolle Dienste.

Seit Göpperts Empfehlung haben wir die Nährzuckerklistiere häufig angewandt und können die guten Erfolge der rectalen Zuckerzuführung in den oben erwähnten Fällen schwer ernährbarer Kinder bestätigen. Unter dem EinfluB der Nährlklysmen führte die zur Leerstellung des Dünndarms erforderliche Hungerdiät selbst bei schweren Atrophikern seltener zum Kollaps als früher. Es scheint sich auch die Reparation dieser Kranken schneller anzubahnen, wenn in den auf die Teepause folgenden Tagen die Brennstoffzufuhr durch 